\title{
DIVERSIDAD Y CONSERVACION DE PRIMATES EN LA RESERVA COMUNAL TAMSHIYACU-TAHUAYO, LORETO, PERU
}

Pablo Puertas *

Richard Bodmer**

Rolando Aquino*

\section{RESUMEN}

El estudio trata sobre la diversidad de los primates y el estado de conservación de la Reserva Comunal Tamshiyacu-Tahuayo. El reporte de catorce especies de primates constituye el mayor registro para unidades de conservación en el Perú. Sin embargo, por efecto de cacería las densidades y biomasas de los cébidos en el área de estudio están siendo drásticamente reducidos, excepto los pichicos, Saguinus mystax y S. fuscicollis. Para contrarrestar la presión de caza, un plan de manejo de fauna silvestre con participación comunitaria viene desarrollándose a fin de asegurar la conservación de los primates y de otros mamíferos en la citada Reserva Comunal.

Palabras claves: Reserva Comunal Tamshiyacu-Tahuayo, diversidad de primates, cébidos, pichicos, presión de caza.

\section{ABSTRACT}

This study presents the diversity of primates and their conservation status in two areas of the Tamshiyacu-Tahuayo Community Reserve. Fourteen primate species were registered, representing the highest diversity reported for a conservation area in Peru. However, due to hunting pressure the densities and biomasses of cebids in the TahuayoBlanco area are being dramatically reduced, this is not occurring with

Centro de Investigación IVITA de la Universidad Nacional Mayor de San Marcos, Aprtado Aéreo 575, Iquitos, Perú.

** Tropical Conservation and Development Program, Latin American Studies 319 Grinter Halll, University of florida, Gainesville, Florida 32611-5531, USA 
callitrichids Saguinus mystax and Saguinus fuscicollis. At this time and experimental mana-gement plan concerning te hunting of mammal is being developed for the conservation of primates population and other mammals.

\section{INTRODUCCION}

La Reserva Comunal Tamshiyacu-Tahuayo (RCTT), establecida por Resolución Ejecutiva Regional № 080-CR-P del 19 de junio de 1991 tiene como propósito desarrollar programas para el aprovechamiento sostenible de los recursos naturales con la finalidad de propiciar mejores oportunidades de vida de la gente local a través de la participación comunitaria.

La RCTT caracterizada por su gran diversidad de fauna y flora silvestre, en su mayoría no están cuantificadas. Los estudios realizados en la RCTT están referidos principalmente a la ecología y manejo de ungulados (Bodmer et al 1988, 1990a, 1990b, 1994a, 1994b, Bodmer 1989), aspectos ecológicos y de comportamiento de los primates, con énfasis en Saguinus mystax y Saguinus fuscicollis (Norconk 1986; Garber 1988; Heymann 1992a). Asímismo se dispone de información preliminar sobre otras especies de primates de tamaño grande como Cacajao calvus y Pithecia monachus (Bartecki \& Bartecki 1990).

Como una contribución al manejo y la conservación de primates en la RCTT, los autores presentan sus resultados obtenidos durante las actividades de censos y registros de caza realizados en dos áreas de la reserva.

\section{MATERIALES Y METODOS}

\section{PERÍODO Y AREAS DE ESTUDIO}

La RCTT tiene una área de 322,500 has. de bosque contínuo conformado predominantemente por hábitats no inundables $(>75 \%)$ ó de tierra firme Bodmer et al. (199a); Puertas \& Bodmer (1993). El área de estudio localizado en la zona del Tahuayo-Blanco esta sometido a una persistente presión de caza, mientras que en la zona del Yavarí Mirí la presión de caza es moderada. 
La mayoría de los habitantes rurales que hacen uso de la Reserva son mestizos conocidos como "ribereños". Dentro sus límites la Reserva no tiene asentamientos humanos permanentes. Par acciones de conservación y manejo ésta Reserva se divide en dos zonas: 1) zona de carácter intangible para la protección de los recursos naturales y 2) zona de amortiguamiento para el uso de subsistencia. Sin embargo en áreas adyacentes a la reserva existen asentamientos humanos permanentes, constituidos por 31 caseríos, que representan una población superior a los 4,500 habitantes. Esta última área no fue oficialmente incorporada, evitando así conflictos por prácticas diversas en el uso de la tierra. No obstante, constituyen una parte fundamental para la incorporación de programas de conservación y desarrollo comunal.

El estudio fue ejecutado en dos áreas de la Reserva, entre junio de 1992 y diciembre de 1993. Las áreas se encuentran en las zonas del : a) Tahuayo-Blanco y b) Yavarí Mirí (Figura 1).

\section{CENSOS Y TECNICAS DE COLECCION DE DATOS}

Para determinar la densidad poblacional de mamíferos en el área del Tahuayo-Blanco de aproximadamente $200 \mathrm{Km}^{2}$ fueron recorridos 120 $\mathrm{km}$ de transectos lineales, mientras que en el area del Yavarí Mirí de aproximadamente $180 \mathrm{Km}^{2}$, fueron recorridos $170 \mathrm{~km}$. Ambas áreas están integradas por bosque contínuo de tierra firma sin interrupción por ríos u otras formaciones ecológicas. Hicimos cinco recorridos de censo por los transectos, cuyas longitudes variaron de 1 a $7 \mathrm{~km}$ dependiendo de las condiciones meteorológicas. Los censos fueron interrumpidos cuando hubo lluvia. La actividad censal fue realizada en los horarios siguientes: 1) por las mañanas entre las 05:30 $\mathrm{h}-11: 00 \mathrm{~h}, 2$ ) por las tardes entre las 15:00-18:00 $\mathrm{h}$ y 3 ) por las noches entre las 19:00 $\mathrm{h}$ a 21:00 h, coincidente principalmente con las horas de mayor actividad alimenticia y de locomoción de los mamíferos diurnos y nocturnos. Al contactar con un grupo de primates, se anotaron la especie, tamaño, hora, actividad y tipo de hábitat. La distancia perpendicular a la trocha del primer animal avistado fue medido en metros. Los estimados de densidad fueron calculados usando la serie de expansión de fourier (Burnhan et al 1980, Brockelman \& Ali 1987), cuya tecnica es una de las más apropiadas para el caso de primates y otros mamíferos en bosques tropicales con tamaño de muestras igual o mayor a 30 
avistamientos (Burnham et al 1980, Ayres 1986). En este estudio el tamaño de muestra para la mayoría de las especies superó los 50 avistamientos. Sin embargo hubieron registros de especies en el rango de 15 a 30 avistamientos, en cuyos casos reportamos densidades relativas.

Los animales fueron identificados por observación directa y comparado con especímenes de las colecciones de la Universidad Nacional de la Amazonía Peruana y Proyecto Peruano de Primatología "Manuel Moro Sommo".

\section{CAZA DE PRIMATES}

Los registros de la caza fueron realizados por personas del lugar quienes fueron debidamente entrenados para el caso. Los registros estaban referidos a la fecha de caza, identificación del animal, sexo, lugar de caza y nombre del cazador.

\section{RESULTADOS}

\section{REGISTRO DE PRIMATES}

Durante los censos, catorce especies de primates fueron registrados en la RCTT (Cuadro 1), que corresponden a 3 especies de Callitrícidos y 11 de Cébidos. Entre los primates congéneres que habitan la Reserva se encuentran Saguinus mystax y S. fuscicollis, Cebus apella y C. albifrons, Saimiri sciureus y $S$. boliviensis. Esta última especie fue reportado por Puertas \& Bodmer (1993). Entre los de tamaño grande figuran Cacajao calvus "huapo rojo", Pithecia monachus "huapo negro", Lagothrix lagotricha "choro", Ateles paniscus "maquisapa" y Alouatta seniculus "coto". También habitan en la reserva Aotus nancymae (A. nancymai) "musmuqui", Callicebus cupreus "tocón colorado" y el mono cebuella pygmaea "leoncito". 


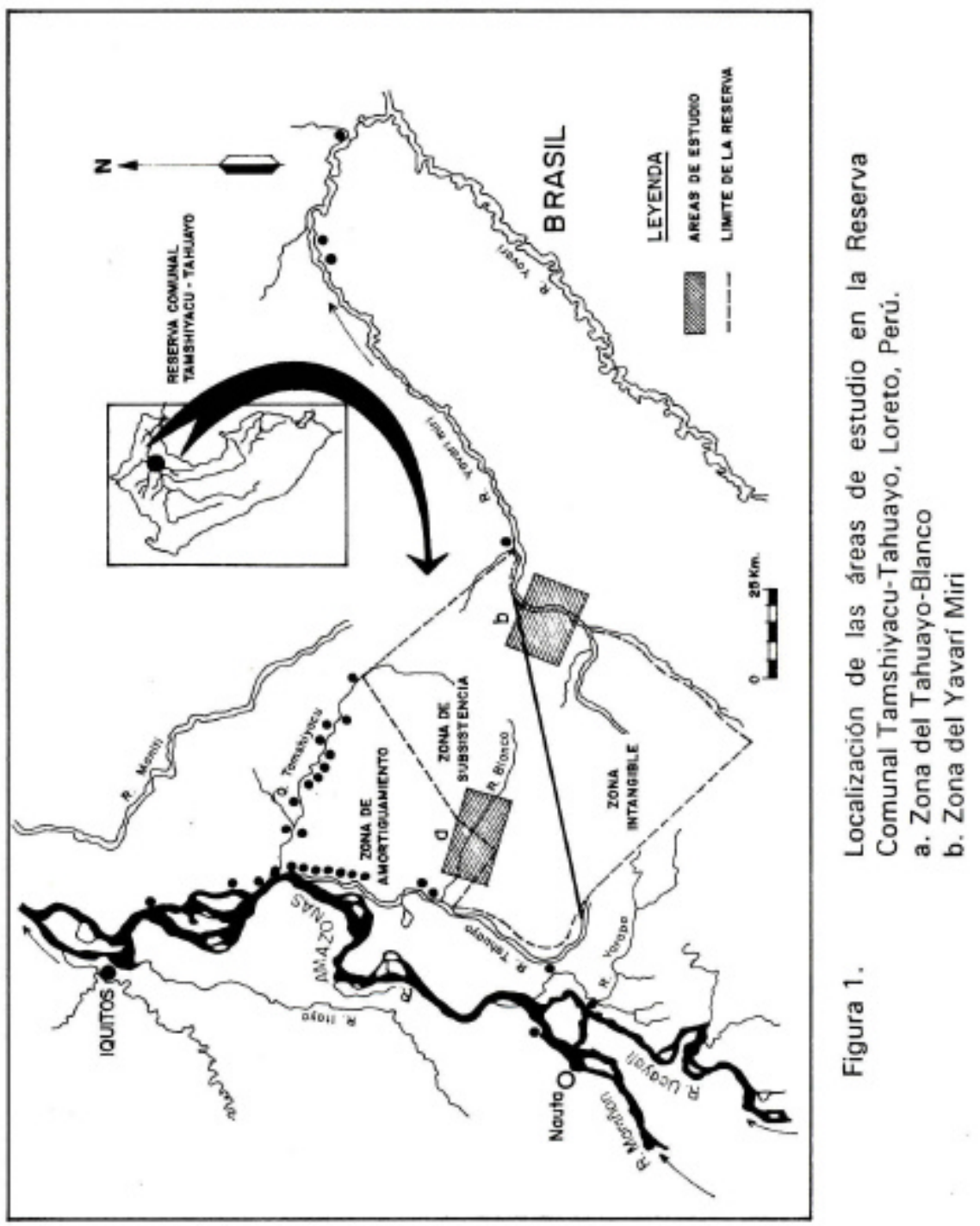




\section{Cuadro 1}

Primates registrados en la Resenva Comunal Tamshiyacu-Tahuayo, noreste del Perú

\section{E S P E C I E}

Callitrichidae

1. Cebuella pygmaea (Spix, 1823)

2. Saguinus fuscicollis (Spix, 1823)

3. Saguinus mystax (Spix, 1823)

Cebidae

4. Aotus nancymae (Poeping, 1826)

5. Saimiri boliviensis (Goeffroy \& Blainville, 1834)

6. Saimiri sciureus (Linnaeus, 1758)

7. Callicebus cupreus (Spix, 1823)

8. Pithecia monachus (Geooffroy, 1812)

9. Cacajao calvus (Spix, 1823)

10.Cebus albifroons (Humboldt, 1812)

11.Cebus apella (Linnaeus, 1758)

12. Ateles paniscus (Linnaeus, 1766)

13. Alouatta seniculus (Linnaeus, 1766)

14. Lagothris lagotricha (Humboldt, 1812)

\section{DENSIDAD POBLACIONAL}

Las densidades estimadas para cada especie están contenidas en el cuadro 2, donde apreciamos que las densidades más altas corresponden a Saimiri sciureus; mientras que la más baja densidad corresponde a Ateles paniscus. 


\section{SITUACION DE LOS PRIMATES}

La alta presión de caza ejercida por los ribereños han disminuido las densidades y biomasa de los cébidos en el área del Tahuayo-Blanco (Cuadro 2). En contraste, la biomasa de los cébidos en el área del Yavarí Mirí fue estimado en $241.6 / \mathrm{km}^{2}$ y representa más del doble de lo estimado para el Tahuayo-Blanco que es de $92.8 / \mathrm{km}^{2}$ (Cuadro 2).

Durante el período de estudio, en una área de $5000 \mathrm{~km}^{2}$ del Tahuayo-Blanco, los ribereños cazaron 323 cébidos (Cuadro 3). Es decir la biomasa extraída de primates alcanzó $948.1 \mathrm{~kg}$. La biomasa de los pichicos en el Yavarí Mirí de $15.5 \mathrm{~kg} / \mathrm{km}^{2}$, y fue similar a la biomasa de los pichicos de Tahuayo-Blanco con $17.4 \mathrm{kmg} / \mathrm{km}^{2}$. Esta similitud de las poblaciones de pichicos sugiere que los bosques del Yavarí Mirí y del Tahuayo-Blanco son similares y las diferencias en la biomasa de cébidos podría ser la resultante de la presión de caza. Es preciso indicar que los callitrícidos son raramente cazados debido a su tamaño pequeño.

\section{DISCUSION}

\section{FACTORES QUE INFLUYEN SOBRE LA DIVERSIDAD BIOLOGICA}

Un total de catorce especies de primates han sido registrados en la RCTT Puertas \& Bodmer (1993). Anteriormente Bodmer et al. (1990a) habían reportado trece especies. La especie adicional corresponde a $\mathrm{S}$. boliviensis, que habita preferentemente los bosques de bajial del Tahuayo-Blanco, mientras que su congénere $\mathrm{S}$. sciureus habita los bosques de colina. 


\section{Cuadro 2.}

Promedios de peso corporal, densidades y biomasas de primates en las dos áreas de estudio de la Reserva Comunal Tamshiyacu-Tahuayo. Las biomasas de las especies fueron calculados multiplicando la densidad de individuos por el promedio del peso corporal. Los promedios de peso corporal fueron según Freese et al. (1982), Aquino \& Encarnación (1986) y Soini (1990).

\begin{tabular}{|c|c|c|c|c|c|}
\hline & Promedio & Tahuayo-B & Blanco & Yavarí M ir & \\
\hline Especies & $\begin{array}{l}\text { Peso corporal } \\
(\mathrm{kg})\end{array}$ & $\begin{array}{l}\text { Densidad } \\
\text { Ind } / \mathrm{km}^{2}\end{array}$ & $\begin{array}{l}\text { Biomasa } \\
\text { Ind } / \mathrm{km}^{2}\end{array}$ & $\begin{array}{l}\text { Densidad } \\
\text { Ind } / \mathrm{km}^{2}\end{array}$ & $\begin{array}{l}\text { Biomasa } \\
\text { Ind } / \mathrm{km}^{2}\end{array}$ \\
\hline
\end{tabular}

Callitrichidae

Saguinus fuscicollis

0.3

21.7

6.5

37.8

11.3

S. mystax

0.5

21.7

10.9

8.4

4.2

Cebidae

A otus nancymae

Saimiri sciureus

Callicebus cupreus

Pithecia monachus

Cacajao calvus

Cebus albifrons

Cebus apella

A teles paniscus

Lagothrix lagotricha
0.5

0.5

0.7

1.1

3.8

1.7

1.9

6.0

4.5
25.0

18.0

2.0

3.5

2.5

5.6

7.7

7.2
2.5

9.0

1.4

3.9

9.5

9.5

14.6

-

32.4
24.0

57.0

2.5

5.0

7.7

7.5

11.3

2.5

25.7
12.0

28.5

1.8

5.5

13.1

28.5

21.5

15.0

115.7

TOTAL

110.2

257.1

Entre otras unidades de Conservación que también cuenta con una gran diversidad de primates resalta el parque Nacional Manú, situado al sur este del Perú, con 13 especies confirmadas y una aún no conformada Terborgh (1983), J anson \& Emmons (1990). Asimismo, hay áreas protegidas en la Amazonía Brasilera que podrían exceder la diversidad de primates reportados en este estudio Rylands \& Bernardes (1989). Sin embargo estas diversidades hasta la fecha no han sido confirmados por observaciones de campo. 
La alta diversidad de mamíferos en bosques de tierra firme entre el Yavarí y el Amazonas se debe probablemente a la combinación de tres factores principales: 1) a refugio del pleistoceno, 2) a la dinámica de los ríos y, 3) a la diversidad florística. Varios autores han sugerido que la regida entre el Yavarí y el Amazonas puede haber contenido uno o dos refugios de pleistoceno Haffer (1969), Prance (1985). Sin embargo, Haffer (1969) limita este refugio a la desembocadura del Yavarí, en cambio Prance (1985) hace referencia de un refugio contínuo comprendido entre el norte peruano y a la Amazonía Ecuatoriana. El argumento de refugios del pleistoceno está únicamente referida hacia aquellas áreas donde se hicieron colectas biológicas Nelson et al (1990). En ese sentido, el Yavarí es una área interesante debido a la escasa coolección de campo existente que permitan aclarar o explicar la hipótesis sobre los refugios del pleistoceno.

La alta diversidad de mamíferos, entre ellos principalmente los priamtes alopatricos en la Amazonía, puede ser el resultado de límites impuestos por la dinámica de los ríos Ayres \& Clutton-Brock (1992). De este modo, los límites impuestos por los ríos Yavarí y el Amazonas podrían haber contribuido a una alta diversidad de primates en la RCTT. Cabe indicar que el río Amazonas limita los rangos geográficos de 39 especies de primates Ayres \& Clutton-Brock (1992). El río Yavarí es considerablemente más pequeño y por ende menos efectivo como barrera geográfica. El Amazonas cambia su curso desde el norte hacia el este antes de llegar a la desembocadura del Yavarí. Así, las distribuciones geográficas de varias especies de primates que ocurren al este del río Yavarí tienen sus límites de distribución occidental y septentrional en la margen izquierda del Amazonas.

Otros de los factores que probablemente ha influenciado la diversidad de mamíferos en la RCTT es la diversidad florística de la A mazonia occidental. La diversidad de árboles en la Amazonía occidental es considerablemente mayor en los bosques del oriente brasilero y las Guyanas Gentry (1988). Además, la diversidad de primates en la amazonia occidental es mayor que el de la cuenca oriental. Ayres (1986) ha sugerido una relación positiva entre la flora y las diversidades de primates al realizar comparaciones entre la amazonia oriental y la ccidental. Indudablemente, la riqueza de primates de la RCTT ha sido influenciado por la alta diversidad florística de los bosques situados entre el Yavarí y Amazonas. Sin embargo, es de mencionar que no solo un factor ha contribuido a la diversidad de prmates observados, sinó una 
combinación de factores y eventos históricos anteriormente mencionados.

Cuadro 3

Presión de caza y biomasa extraída de primates en la Reserva Comunal Tamshiyacu-Tahuayo

$\begin{array}{cc}\text { ESPECIES } & \text { NUMERO DE ANIMALES } \\ \text { CAZADOS } & \text { EXTRAIDA } \\ & (\mathrm{kg} .)\end{array}$

Callitrichidae

Cebuella pygmaea

Saguinus sp

1

11

4.0

9.0

76.0

57.0

23.0

20.0

46.0

8.0

22.00

58.0
0.1

4.4

Cebidae

Saimiri sciureus

Pithecia monachus

Cacajao calvus

Cebus albifrons

Cebus apella

A teles paniscus

Alouatta seniculus

Lagothris lagotricha

TOTAL

335.0

948.1

3.2

6.3

72.2

85.5

87.4

46.0

115.0

48.0

132.0

342.0

* Los promedios de peso corporal únicamente en adultos fueron obtenidos de Fontaine (1981), Freese et al (1982), Aquino \& Encarnación (1986) y Soini (1990). 


\section{CONSERVACION DE LA DIVERSIDAD BIOLOGICA}

Los esfuerzos para relevar la importancia para la conservación de la diversidad biológica están dirigidos prioritariamente hacia aquellas especies endémicas Western \& Pearl (1989). Sin embargo, debe ser igualmente importante considerar a la naturaleza endémica de las especies a nivel de ecosistemas funcionales Solbrig (1991).

El manejo de los bosques de la RCTT es una necesidad propia para la conservación de las especies. Una de las acciones más urgentes requiere el cese de la caza de primates y de otros mamíferos de tamaño grande como Tapirus terrri "tapir ó sachavaca" debido a la contínua explotación están haciendo disminuir a las poblaciones de esas especies.

La presión de caza en el Tahuayo-Blanco parece ser un resultadoo de su proximidad a la ciudad de lquitos, ya que puede llegarse en bote enn aproximadamente 10 horas de viaje. Como coonsecuencia de cazadores de esta área están influenciados primariamente por el valor de la carne de monte en los mercados de Iquitos Bodmer (1990a). Los primates también son expendidos coomo carne de monte. Sin embargo los cazadores prefieren cazar ungulados y roedoores de tamaño grande debido a su mayor aceptación y cotización del mercado, siendo la venta de tales animales al contado. Los primates y otros mamíferos de menor tamaño soon utilizados en la alimentación durante la temporada de cacería a fin de substituir la pérdiida económica que los cazadores incurriríán si consumieran la carne convallor coomercial de los mamíferos con mayor tamaño.

Una plan de manejo sobre la caza de subsistencia en la RCTT viene siendo desarrollado para el área del Tahuayo-Blanco. El modelo de manejo coonsidera la biología poblacional de las especies cazadas, el análisis económico de los cazadores y las limitaciones sociales del manejo de la caza en el área Bodmer (1994). El modelo para el TahuayoBlanco establece que los beneficios de la carne de monte serían derivados únicamente de los machos artiodáctilos y roedores de tamaño grande. En cambio, para el consumo con fines de subsistencia las hembras artiodáctilos y los roedores de tamaño grande. Estamos convencidoos que la carne de las hembras artiodactilos y roedores de tamaño grande usados para coonsumo de subsistencia substituirán la carne perdida de los primates u otros mamíferos de tamaño pequeño. El plan es que en el futuro los cazadores no tendrán el estímulo de cazar primates ni tapir para consumo, mientras haya disponibilidad de la carne de los artiodáctilos hembras y roedores de tamaño grande. 


\section{CONCLUSIONES}

Los bosques de la Reserva Comunal Tamshiyacu-Tahuayo presentan la mayor diversidad de primates reportados a la fecha para unidades de conservación en el Perú.

Las densidades y biomasas de los primates cebidos en el área del Tahuayo-Blanco vienen siendo drásticamente reducidas por efectos de la cacería.

El manejo adecuado de los bosques de la Reserva Comunal Tamshiyacu-Tahuayo es una necesidad primordial para la conservación de la diversidad de primates y de otras especies.

Un plan de manejo de la caza de subsistencia en la Reserva Comunal Tamshiyacu-Tahuayo debe ser desarrollado para el área del Tahuayo-Blanco, el cual tome en cuenta la biología poblacional de las especies cazadas, el análisis ecoonómico costo-beneficio de los cazadores y las limitaciones sociales del manejo de la caza.

\section{AGRADECIMIENTO}

Expresamos nuestro especial agradecimiento al Proyecto Peruano de Primatología "Manuel Moro Sommo", Consejo Nacional de Ciencia y Tecnología (CONCYTEC) y a la Asociación para la Conservación de la A mazonía (ACA) en la persona del Sr. J ames Penn por el apoyo técnico, financieron y logístico proporcionado. Este estudio fue realizado coomo parte del programa: "Manejo de Fauna Silvestre para la Conservación de los Bosques Amazónicos" (Carta de Entendimiento suscrito entre el Dr. Richard E. Bodmer del Tropical Conservation and Development Program, Latin American Studies, University of Florida, Gainesville, USA y la EE Iquitos/CI IVITA, representado por el Dr. Enrique Montoya). 


\section{BIBLIOGRAFIA}

AQUINO, R., F. ENCARNACION. 1986. Population structure of Aotus nancymai (Cebidae: Primates) in Peruvian Amazon Lowland forest. A merican J ournal of Primatology, 11: 1-7.

AQUINO, R. 1988. Preliminary survey on the population densities of Cacajao calvus ucayali. Primate Conservation, 9: 24-26.

AYRES, J. 1986. Uakaris and Amazonian flooded florest. Tesis para optar el grado de Ph. D., University of Cambridge

AYRES, J., T. CLUTTON-BROCK, 1992. River boundaries and species range size in amazonian primates. American Naturalist. 140: 531537.

BARTECKI, U., E.W. HEYMANN, 1988. Observations on predatory behaviour of a young saki monkey, Pithecia hirsuta (Platyrrhini: Cebidae). Priamte Report, 20: 29-33.

BODMER, R., 1989. Frugivory in amazon ungulates. Tesis para optar el grado de Ph. D., University of Cambridge.

-, 1994. Managing wildlife with local communities in the Peruvian A mazon: The case of the Reserva Comunal Tamshiyacu. En: Natural Connections, Perspectives in Community-based Conservation (D.Western, R.M. Wright y Sh. C. Strum, ed) pp. 113-134. Island Press, Washington D.C.

BODMER, R., L. MOYA y T. FANG, 1988. Ungulates management and conservation in the peruvian amazon Biology Conservation, 45: 303-310.

BODMER, J. , J. PENNN, T. FANG y L. MOYA, 1990a. Mangement programmmes and protected areas: the case of the Reserva Comunal Tamshiyacu-Tahuayo, Perú. PARKS, 1(1):21-25.

BODMER, J., N. BENDAYAN, L. MOYA y T. FANG, 1990b. Manejo de ungulados en la amazonía peruana: análisis de caza de comercialización. Boletín de Lima, 70:49-56.

BODMER, J ., P. PUERTAS, L. MOYA y T. FANG, 1994a. Evaluación de las poblaciones de tapir de la amazonía peruana. Fauna en camino de extinción. Boletín de Lima, 88: 33-42. 
BODMER, J., T. FANG, L. MOYA y R. GILL, 1994b. Managing wildlife to conserve amazonian forest: Population biology and economic considerations. Biological Conservation, 67: 29-35.

BROCKELMAN, W. y R. ALI, 1987. Methods of survering and sampling forest primate population. En primate conservation in tropical rainforests. C.W. Marsh and R.A. Mittermeier (eds), Alan R. Liss, New York, pp. 23-62.

BURNHAM, K., D. ANDERSON, L. LAAKE, 1980. Estimation of density from line transect sampling of biological populations. Wildlife Monographs. pp. 72.

FONTAINE, R. 1981. The Uakaris, Genus Cacajao. En: Ecoology and Behavior of Neotropical Primates. Vol 1. Academia Brasileira de Ciencias, Río de J aneiro (A.F. Coimbra-Filho y R.A. Mittermeier eds.) pp. 443-493.

FREESE, CH., P. HELTNE, N. CASTRO y G. WHITESIDES, 1982. Patterns and Determinants of Monkey Densities in Peru and Bolivia, with Notes on Distributions. International J ournal of Priamtology, 3(1): 53-90.

GARBER, P., 1988. Diet, foranging patterns, and resoource defense in a mixed species troop of Saguinus mystax and Saguinus fuscicollis in Amazonian Peru. Behaviour, 105: 18-34.

GENTRY, A. 1988. Tree species richness of upper amazonian forests. Proceedings of the national academy of science, 85: 156-159.

HAFFER, J. 1969. Speciation in amazonian forest birds. Science, 165: $131-37$.

HEYMANN, E.W., 1992a. Associations of tamarins (Saguinus mystax and Saguinus fuscicollis) and double-toothed kite (Harpagus bidantatus) in Peruvian Amazonia. Folia Primatológica, 59: 51-55.

1992b. The red uakari (Cacajao calvus ucayalii): some field observations and speculations on a little-known species. Primate Eye, 47: 6-7. 
HEYMANN, E.W., y U. BARTECKI, 1990. A young saki monkey, Pithecia hirsuta, feeding on ants, Cephalotus atratus. Folia Primatologica. 55: 181-184.

J ANSON, C. y L.EMMONS, 1990. Ecological structure of the nonflying mammals community al Cocha Cashu Biological Station. Manu National Park Peru. En: Four Neotropical Rainforest (A.H. Gentry, ed.) pp. 314-318. New Haven: Yale University Press.

NELSON, B., C. FERREIRA, M. DA SILVA y M. KAWASAKI, 1990. Endemism centres, refugia and botanical collection density in Brazilian amazonia. Nature, 345: 714-716.

NORCONK, M. 1986. Interactions between primate species in a neotropical forests: mixed-species troops of Saguinus mystax and Saguinus fuscicollis (Callitrichidae). Ph. D. Dissertation, University of California, Los Angeles.

PRANCE, G. 1985. The changing forests. In: Key environments: Amazonia. G.T. Prance and T.E. Lovejoy, eds. Pergamon Press, Oxford, pp. 146-165.

PUERTAS, P., R. BODMER, 1993. Conservation of a high diversity primate assemblage. Biodiversity and Conservation 10: 56-62.

SOINI, P., 1990. Ecología y dinámica poblacional de pichico común Saguinus fuscicollis (Callitrichidae, Primates). En : La Primatología en el Perú. Investigaciones Primatológicas. Propaceb, Lima, pp. 202 253.

SOLBRIG, O., 1991. Fron genes to ecosistems: A research agenda for biodiversity. Cambridge Mass. IUBSS-SCOPE-UNESCO.

TERBORGH, J . 1983. Five New World Primates: A Study in Comparative Ecology. Princeton: Princeton University Press.

WESTERN, N. y M. PEARL, 1989. Conservation for the twenty-first century. Oxford University Press. Oxford. 